\title{
Probabilistic evaluation approach for electrical connector mating: An empirical study on automotive electronic connectors
}

\author{
Chunghun HA*, Hong-Bae JUN* and Changsoo OK* \\ *Department of Industrial Engineering, Hongik University \\ 94 Wausan-ro, Mapo-gu, Seoul 04066, Korea \\ E-mail: okcs@hongik.ac.kr
}

Received: 5 December 2016; Revised: 8 October 2017; Accepted: 6 December 2017

\begin{abstract}
Half engagement is a state that metal terminals are mated, but mechanical housings are not mated completely. When a connector is in such state, the possibility of connector separation is high even for a small external disturbance. It may cause a serious malfunctioning to the parent product. During manufacturing, however, it is difficult to detect the state because electrical functions still work properly. Lack of feeling of mating is known as the primary cause of the state. Normally the feeling of mating is unmeasurable. Therefore, it is necessary to quantify the feeling of mating to prevent the half engagement. To this end, in this paper, we have proposed a new evaluation approach for the half engagement prevention. We have adopted a probabilistic structure based on reliability theory and applied the logistic regression method as a scoring tool. Moreover, we have introduced two new evaluation factors to increase the accuracy of state detection. We have verified and compared the performance of our proposed approach to that of the conventional approach which has a deterministic structure. Throughout extensive experiments and analyses with real automotive electronic connectors, we have confirmed that the proposed approach is superior to the existing one in terms of the accuracy of state detection.
\end{abstract}

Keywords : Electrical connector, Half engagement, Reliability structure, Logistic regression

\section{Introduction}

As the portion of electronic parts in automotive increases, the importance of wiring harness including connectors is increased rapidly. Most automotive companies employ various electronic systems competitively to achieve a competitive advantage (Leen and Heffernan 2002). The electronic devices include ignition system, trans-axle control system (TACS), engine management system (EMS), anti-lock brake system (ABS), global positioning system (GPS), and automatic temperature control system (ATCS) (Denton 2017). These devices are wired-connected to an electric source for power and other devices for communication. In these connections, electrical connectors play a critical role in connecting wire-to-device and device to device. As shown as Fig. 1-a), an electrical connector is composed of a pair of half-connectors: a male and a female (Mroczkowski 1998). Each half-connector consists of a plurality of metal terminals connected to electrical wires and a resin housing which provides room for the terminals and interlocks the male and the female connectors mechanically. 


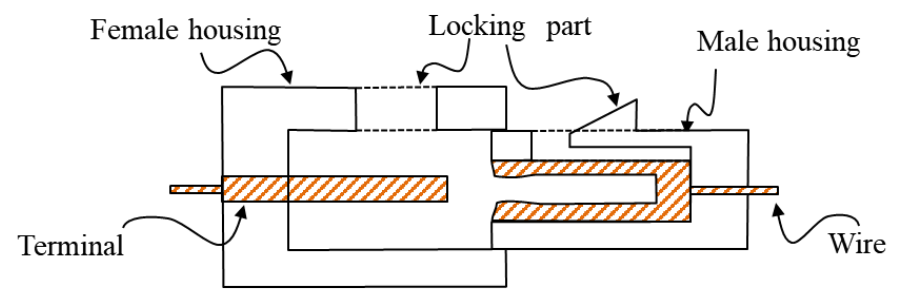

a) Cross sectional view of a typical electronic connector

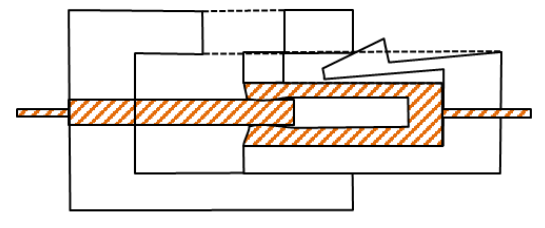

b) Half engagement state

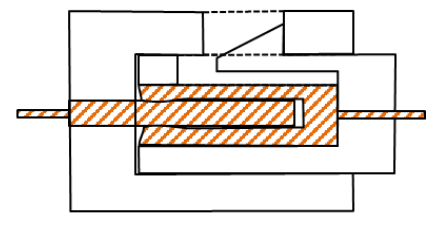

c) Full engagement state

Fig. 1 A typical electronic connector and engagement states

Secure mating between the half-connectors is required for proper functioning of the devices. The mating process is composed of two consecutive steps: the inter-terminal mating for electrical connection and the inter-housing mating for mechanical interlocking. If the both mating operations are perfect (Fig. 1-c)), we call the state as the full engagement. Otherwise, the mating is incomplete (Fig. 1-b)), which leads to the half engagement or the failed engagement. Half engagement is the state that the inter-terminal mating is complete, but the inter-housing mating is imperfect (Kubota and Toi 2009). Design errors, manufacturing faults, human errors, uncomfortable operation posture, and any combination of them may cause the half engagement. In this state, even ordinary vibration or unexpected small force can unfasten the connector mating; consequently, it may cause a serious malfunctioning at the parent product. In an automotive company, an internal report tells that about $20 \%$ of field claims related to automotive electronics are connector problems, where about 50\% among them are half engagements. Rework and field claim increase manufacturing costs. The failed engagement is the state that any of the terminals fails to connect to its counterpart regardless of the inter-housing mating. It mainly occurs due to the defect, deflection, absence, or corrosion of the terminal. Early detection of the failed engagement is critical for productivity and safety (Huang et al. 2010). A well-designed electrical test can find the failed engagement easily. On the contrary, it is difficult to discern the half engagement on inspection or test because a connector in the half engagement state is still electrifying and its appearance looks like perfect.

Every connector generates visual, audible, and haptic signals during a mating process. However, if the signals are vague, a worker may misperceive the mating status and, consequently, it may result in the half engagement by aborting the process en route. An alternative to prevent the half engagement is to design a new connector (Kashiyama and Suzuki 2004; Kashiyama 1999; Kozono and Yamamoto 2005; Yagi et al. 1997). However, these advanced connectors are often infeasible to replace existing connectors due to large insertion force, large volume, lack of compatibility, and high unit cost. Another alternative is to reinforce the assessment of connectors, hence to avoid the use of connectors that have a high probability of the half engagement. It requires quantification of the feeling of mating, but there is no generally acceptable measure for it.

Kubota and Toi ${ }^{(2009)}$ have proposed a deterministic assessment method for the feeling of mating. They considered three key factors; the maximum insertion force, the loudness level of the locking sound, and the force drop rate. In their method, an evaluator scored the amount of each factor according to pre-specified criteria, and then, they calculated the total score of the feeling of mating by summing the individual scores and adjusting workability scores. The total score was used as a measure of the possibility of the half engagement. Notwithstanding the pioneering work, their method has two severe weaknesses. First, they did not provide any empirical verification and validation, which are the most critical steps for developing an evaluation method. Second, the total score could be upside-down. The feeling of mating is not additive. In other words, a strong factor could give a clearer signal than several moderate factors do for complete mating. Thus, a simple summation of individual scores could not reflect this characteristic.

The main objective of this paper is to propose an improved connector assessment methodology from the 
perspective of the half engagement prevention. We have adopted a probabilistic structure based on reliability theory and applied the logistic regression method as a scoring tool. Moreover, we have introduced two new evaluation factors to increase the accuracy of state detection: insertion force profile and visual detection devices. To obtain sufficient data, we have conducted extensive experiments with real automotive connectors. We have also verified and validated the performance of the proposed method via some parametric correlation analysis and nonparametric distance measures.

This paper is organized as follows. In Section 2, the previous evaluation approach of Kubota and Toi (2009) has been introduced and discussed. Section 3 and Section 4 describe the detailed explanations of the two new additional factors and the probabilistic aggregation structure, respectively. Section 5 explains how to perform the extensive experiments and conduct logistic regression for each factor to obtain the probability of half engagement. The comparison of the performance of the proposed to the previous approach is carried out for the coefficients of correlation and nonparametric distance measures in Section 6. Finally, Section 7 states the discussion and conclusions of this paper.

\section{The connector evaluation approach of Kubota and Toi ${ }^{(2009)}$}

Kubota and Toi (2009) introduced a pioneering research for quantification of the feeling of mating. They considered three key factors, which are the maximum insertion force, the force drop rate, and the loudness of locking sound.

\section{- Maximum insertion force}

A typical mating process consists of four phases: starting, sliding, releasing, and completion (Huang, Fukuda, and Matsuno 2008). An insertion force profile, as shown in Fig. 2, is a temporal composition of those phases. In general, the insertion force is measured by a force gauge with a constant speed $(50 \mathrm{~mm} / \mathrm{min})$ and a given sampling rate $(200 \mathrm{~Hz})$. The maximum insertion force $\left(F_{\max }\right)$ is defined as the largest insertion force before completion, and the stroke length $\left(L_{s}\right)$ is defined as the length from the first $10 \mathrm{~N}$ point to $F_{\text {max }}$.

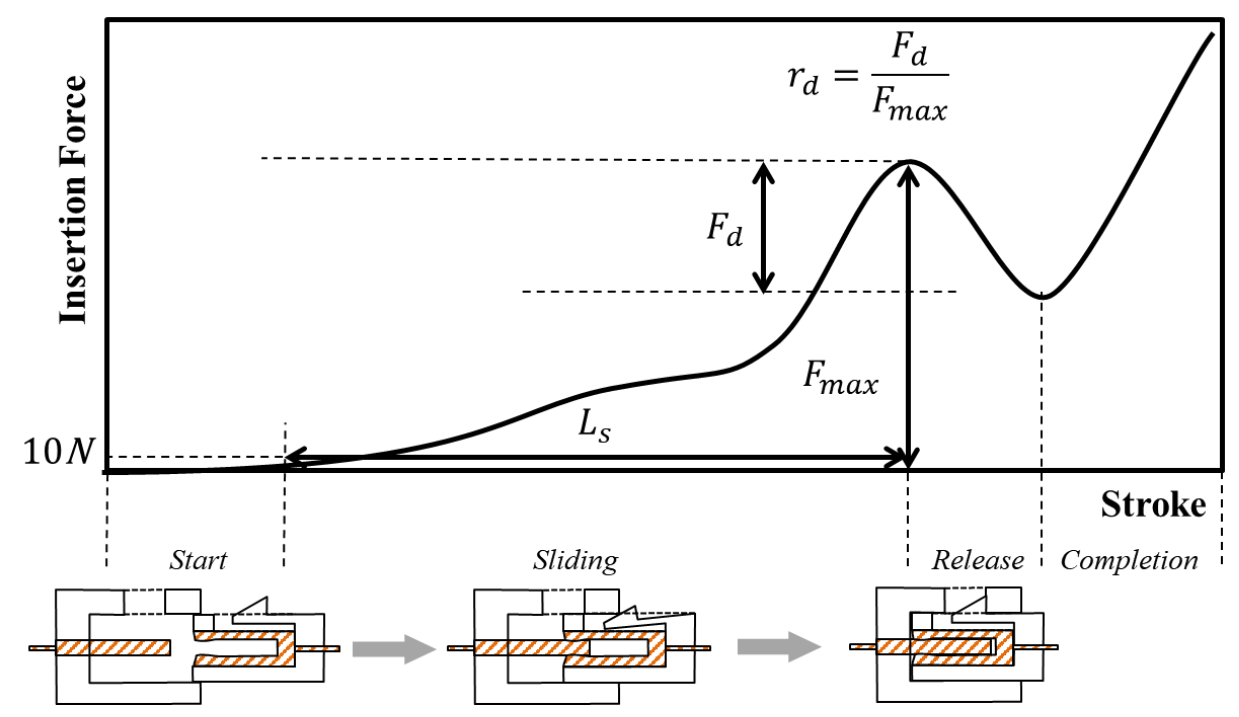

Fig. 2 Standard insertion force profile

\section{- Force drop rate}

When a connector is mating, it generates a haptic signal at the worker's hand, the so-called feeling of force drop. It can be a sign of the completion of the connector mating. A variety of factors such as the diameter of the terminal, the number of terminals, the maximum insertion force, the magnitude of force drop, and the force drop rate, can affect the feeling of force drop. However, in our experiments, the only statistically important factor was the force drop. In the 
insertion force profile (Fig. 2), the force drop $\left(F_{d}\right)$ is defined as the force gap between $F_{\text {max }}$ and the coming through, and the force drop rate $\left(r_{d}\right)$ is defined as the ratio of $F_{d}$ to $F_{\text {max }}$, i.e. $r_{d}=F_{d} / F_{\max }$.

\section{- Loudness of locking sound}

When the locking part of the housing is mating, a clear locking sound is generated. During a mating operation, workers can recognize the completion of mating through the sound. The sound is clearer than the visual and the haptic signals. The visual detection is effective only when workers observe the appearance of the connector carefully. During the mating process, connectors are often invisible, and workers usually do not observe the connectors carefully. The haptic signal becomes dull if the worker is wearing thick gloves. On the other hand, the locking sound has no serious attenuation factors. Even under factory noise, the locking sound can be detectable by workers. Moreover, since the inter-housing mating comes after the inter-terminal mating, the occurance of the locking sound indicates that the full engagement is completed.

With above factors, Kubota and Toi (2009) have proposed the total score of feeling of mating $P_{\text {total }}$ of Eq. (1), where $C_{s}$ is the coefficient of stroke, $P_{f}$ is the insertion force base point, $P_{d}$ is the force drop point, $P_{a}$ is the audible feedback point, and $P_{\propto}$ is adjustable point related to workability, which are determined by the specified criteria in Table 1 .

$P_{\text {total }}=C_{s} P_{f}+P_{d}+P_{a}+P_{\propto}$

Table 1. Criteria of the evaluation system of Kubota and Toi (2009)

\begin{tabular}{c|c|c|c|c|c|c|c}
\hline \hline \multicolumn{3}{c|}{$\begin{array}{c}\text { insertion force point } \\
C_{s} P_{f}\end{array}$} & \multicolumn{2}{c|}{$\begin{array}{c}\text { force drop point } \\
P_{d}\end{array}$} & \multicolumn{2}{c}{ audible feedback point } \\
\multicolumn{1}{c|}{$C_{s}$} & $L_{s}[\mathrm{~mm}]$ & $P_{f}$ & $F_{\max }[N]$ & $P_{d}$ & $r_{d}$ & $P_{a}$ & $A_{l}[d B]$ \\
\hline 1 & $L_{s}<4.5$ & 4 & $F_{\max }<45$ & 4 & $0.2 \leq r_{d}$ & 4 & $70 \leq A_{l}$ \\
\hline 0.75 & $4.5 \leq L_{s}<5.5$ & 3 & $45 \leq F_{\max }<55$ & 3 & $0.1 \leq r_{d}<0.2$ & 3 & $65 \leq A_{l}<70$ \\
\hline 0.5 & $5.5 \leq L_{s}<6.5$ & 2 & $55 \leq F_{\max }<65$ & 2 & $0.05 \leq r_{d}<0.1$ & 2 & $55 \leq A_{l}<65$ \\
\hline 0.25 & $6.5 \leq L_{s}$ & 1 & $65 \leq F_{\max }<70$ & 1 & $r_{d}<0.05$ & 1 & $A_{l}<55$ \\
\hline & linear & & linear & & nonlinear & & nonlinear \\
\hline
\end{tabular}

The insertion force point $C_{S} P_{f}$ becomes larger as $L_{s}$ is shorter and $F_{\text {max }}$ is smaller, that is, the smaller the workload, the higher the score. The force drop point $P_{d}$ increases nonlinearly as the magnitude of the force drop rate $r$ increases. The audible feedback point $P_{a}$ becomes larger as the corresponding loudness level of locking sound increases. Lastly, the adjustable point $P_{\propto}$ is assigned $0,-1$, or -2 points in consideration of the size of working space for hands, anchorage strength of the female connector, and visibility of the connector. For the detailed explanation, refer to Kubota and Toi (2009).

The assessment system of Kubota and Toi (2009) has several drawbacks. First, they did not verify the effectiveness of the method with empirical data, which is critical when developing a new assessment method. They only suggested how to apply the procedure in practice without any empirical results. Second, the total score can be the opposite of the actual feeling of mating. Addition is not valid for the feeling. In other words, adding several types of faint feeling of matings does not give a certainty of the full engagement. For example, let us consider two connectors $\mathrm{A}$ and B with following values: 1) A: $\left.C_{s}=1, P_{f}=3, P_{d}=2, P_{a}=2, P_{\propto}=0,2\right) \mathrm{B}: C_{s}=1, P_{f}=1, P_{d}=1, P_{a}=4, P_{\propto}=$ 0 . The total score of the connector $\mathrm{A}$ is 7 , and that of the $\mathrm{B}$ is 6 . Therefore, we can conclude that the A provides better feeling of mating than the B does. However, in our experience, most operators sense that the feeling of mating of the B is better, because locking sound of the B is clearer than the A. Third, there is no explanation on how to determine the criteria in Table 1. Since the criteria are critical to calculate the score of feeling of mating, clear and definite explanations are required. Forth, they considered the importance of factors equally. According to Table 1, the maximum value of the factors is the same. This means that the influence of each factor on the total score is the same. 
We disagree with this and argue that the influence of each factor should be set differently. Finally, the meaning of the total score is ambiguous. The $C_{s} P_{f}$ and $P_{\propto}$ are relevant to workability and the $P_{d}$ and $P_{a}$ are the factors related to feeling of mating. Since the two types of incompatible factors coexist in a single indicator, the application of the total score is limited.

\section{Two new key factors}

Through experimentation, we found that two factors overlooked by Kubota and Toi (2009) are critical to feeling of mating: insertion force profile and visual detection device. The details of these factors are as follow:

\subsection{Insertion force profile}

In Fig. 2, we presented an insertion force profile of a standard connector. However, every connector has its innate profile. It varies depending on starting locations of terminal insertion, latch shape of housing, materials of terminals and housing, diameter and number of terminals, and type of connector. We have measured insertion force profiles of 76 actual automotive connectors and could classify them into four types as shown in Fig. 3.
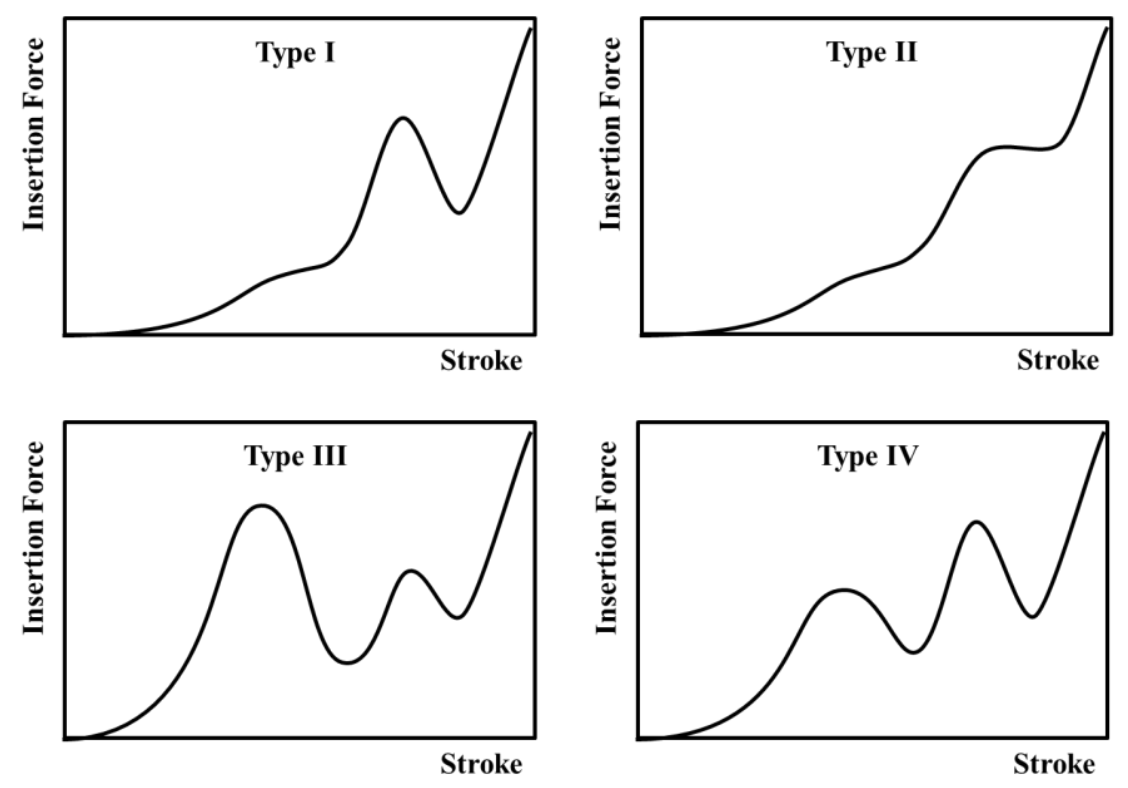

Fig. 3 Four types of insertion force profile

\section{- Type I}

Type I is a standard insertion force profile with a single peak. This type of connector obviously has a force drop. If the force drop rate is large enough, the worker can clearly feel the force drop in his hand during mating.

\section{- Type II}

The insertion force profile of type II connector is similar to one of Type I connector, but has no peak. This type of profile frequently occurs on the connector with large frictional force. The diameter and the number of terminals are the main causes of the friction. Although the force drop rate is almost zero, this type of connector can still generate a faint feeling of the force drop.

\section{Type III}


Type III has dual peaks in the insertion force profile, in which the first peak should be higher than the second one. The first peak occurs due to sliding of the locking part in the housing and the second one due to insertion of terminals. Inertia-lock connector belongs to this type of connector, which is a special connector that enforces mating of terminals using the strong inertia generated by a locking part. If mating speed is fast enough, this type of profile is ideal because it induces full engagement. On the other hand, this type of connector can provide the worker with a fake signal of complete engagement because it generates the force drops twice. In addition, its large maximum insertion force makes the worker more fatigued.

\section{- Type IV}

Type IV has two peaks like the type III, but the second peak is lower than the first one. The insertion of terminals makes the first peak, and sliding of locking part in housing generates the second. This type of connector has the greatest probability of half engagement because force drop occurs twice regardless of the mating speed.

\subsection{Visual detection device}

The visual detection device is an indicator that provides a visual signal of completion of mating. The devices include a guideline, housing hole, and color difference. These visual detection devices have not traditionally been considered because workers do not observe carefully those during mating process. Nonetheless, through education, these devices can give workers additional confidence in the completion of secure mating.

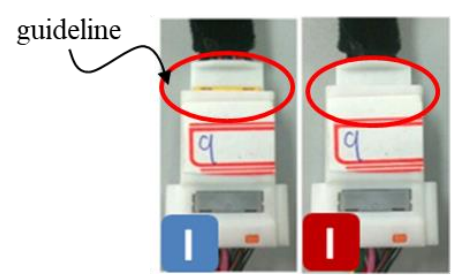

a) Guideline

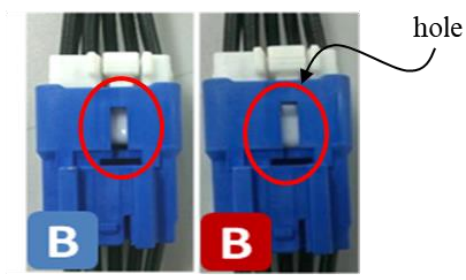

b) Housing hole
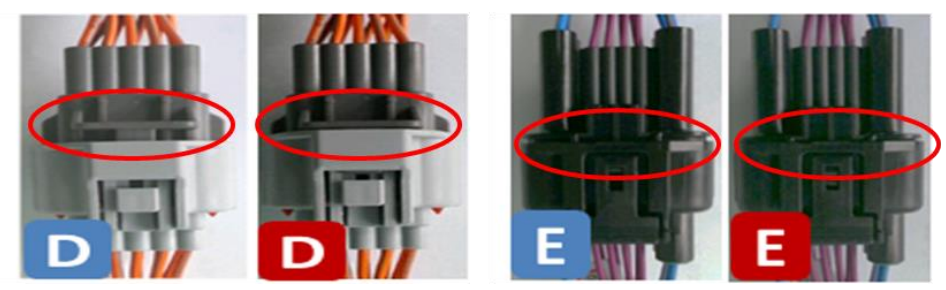

c) Color difference

Fig. 4 Examples of the visual detection devices (left: half engagement state, right: full engagement state)

Fig. 4 shows the examples of the visual detection devices. In each figure, the left-side image depicts a state of half engagement, and the right-side a state of full engagement. The guideline is a yellow indicator as shown in Fig. 4-a). The original purpose of this device is to securely fasten the terminal to the housing, but as shown in the figure, it can also be used to confirm the engagement state. The housing hole is a hole in the female connector as shown in Fig. 4-b). If observation of the worker is ensured, it can also be used as an auxiliary signal to confirm the engagement state of the connector. The color difference between male and female connectors of Fig. 4-c) can also increase the possibility of detecting half engagement. The effectiveness of the devices will be verified in detail in Section 5.1.

\section{The proposed probabilistic evaluation approach}


This section explains a new connector assessment approach to quantify the probability of full engagement. Instead of a simple summation of sub-scores, we employed a probabilistic relationship among the factors. The feeling of mating consists of factors that represent auditory, visual, and tactile feelings, which are visual detection devices, locking sounds, and force drops, respectively. The measure of feeling of mating is the probability of perception of complete engagement (full engagement state). Since the metric is probability, the effect of each factor on the perception probability is not additive. One sufficiently strong factor contributes more to the perception probability than several medium magnitude factors. This probabilistic relationship can be interpreted as a parallel structure of reliability theory (Kuo and Zuo 2003; Elsayed 2012). Fig. 5 is a reliability block diagram showing the probabilistic relationship between the three components of feeling of mating (Lee and Ha 2014). Throughout this paper, we assume the following assumptions: 1) the connector has only two mating states, the full and the half engagements; 2) the failed engagement only occurs from defects of the connector; 3) there is no follow-up activities after detecting a half engagement; 4) the occurrence of locking sound or force drop imply completion of engagement.

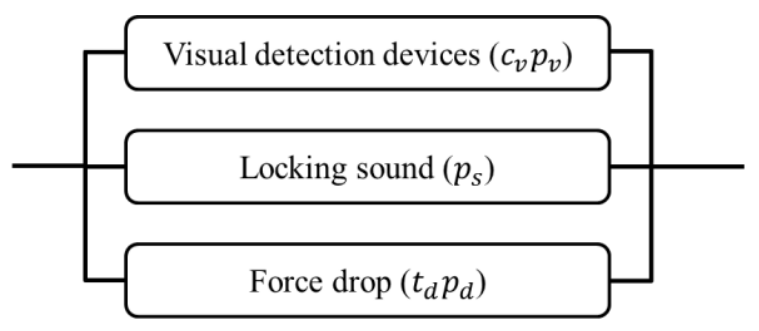

Fig. 5 Reliability block diagram of the perception probability of complete engagement

The perception probability of complete engagement $p_{C E}$ can be calculated by the Eq. (2), where $c_{v}$ is a visibility coefficient which is 1 if the connector under mating is visible to a worker without any special working postures such as bending, leaning, and turning, otherwise, $0 ; p_{v}$ is the probability of distinguishing an engagement status correctly through the visual detection devices; $p_{s}$ is the perception probability of the locking sound; $p_{d}$ is the perception probability of the force drop. The $p_{d}$ should be accompanied by the type of insertion force profile $t_{d}$ defined in Section 3 due to their relevancy.

$p_{C E}=1-\left(1-c_{v} p_{v}\right)\left(1-p_{s}\right)\left(1-t_{d} p_{d}\right)$

The $p_{C E}$ of an ordinary connector is normally very close to one, so the value is indiscriminative. To increase sensitivity, we recommend a sigma level of $p_{C E}$, which is a popular measure in Six Sigma (Pyzdek and Keller 2014). The measure is familiar to practitioners, so more practical than probability. The sigma level of $p_{C E}$, i.e. $Z_{C E}$ can be calculated by the Eq. (3), where $\Phi^{-1}(\cdot)$ denotes the inverse function of the cumulative Standard Normal distribution. Since low $p_{C E}(\leq 0.5)$ implies quite faint feeling of mating, we assume that $p_{C E}$ should be larger than 0.5 , i.e., $Z_{C E}$ is positive.

$Z_{C E}=\Phi^{-1}\left(p_{C E}\right)$

The proposed probabilistic assessment has several advantages over the previous one. First, it is flexible. Even if a factor is added or removed, there is no need to change the evaluation system itself. For example, suppose further consideration of workability factors. Not enough hand space and uncomfortable working posture prevent worker from mating connector. Those can decrease the probability of complete engagement despite high $p_{C E}$. These factors are not complementary and independent, so they are linked to the existing assessment structure as series, as shown in Fig. 6. Then, the probability of complete engagement should be modified as the Eq. (4), where $p_{h}$ and $p_{m}$ are the probability of complete engagement owing to the hand space and the posture, respectively. Despite the change of the equation, since the measure is still probability and the range and the value are retained, further steps in the following sections can be applied without loss of generality. 


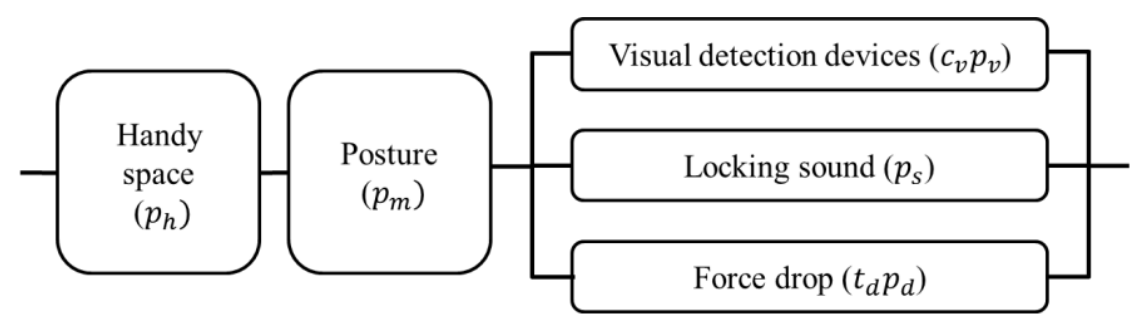

Fig. 6 Reliability block diagram of connector mating considering workability factors

$p_{C E}=p_{h} p_{m}\left[1-\left(1-c_{v} p_{v}\right)\left(1-p_{s}\right)\left(1-t_{d} p_{d}\right)\right]$

Second, the proposed approach can automatically adjust importance of the factors. As we mentioned in Section 2, the importance of each factor is not equal. Since the proposed assessment measures and estimates each factor as probability, the importance is adjusted when the $p_{C E}$ is calculated. Third, the proposed assessment provides continuous value within the range of 0 to 1 as a result because the metric is continuous. On the other hand, the final score of Kubota and Toi (2009) is a positive integer. The continuous result can improve the precision and sensitivity of the assessment. Finally, the proposed assessment is also helpful to optimize and improve the connector design. Suppose that a connector designer has several alternatives for the visual detection devices, sound pressure level of locking sound, and insertion force profile in a connector design. Then, finding the optimal combination of the alternatives to maximize the $p_{C E}$ under a specified volume and cost constraints is a well-known reliability allocation problem (Ha and Kuo 2008; Kuo and Zuo 2003).

\section{Estimation of factor probability}

To apply the proposed approach, we should determine the parameters and probabilities of factors $\left(c_{v}, t_{d}, p_{a}, p_{v}, p_{s}, p_{d}\right)$. Extensive experiments have been conducted to obtain empirical data of actual connectors, then performed binary logistic regression analyses to estimate the probabilities (Menard 2002). The ordinary regression is inappropriate to our model because the dependent variables are dichotomous, i.e. success or failure of perception.

\subsection{Logistic regression analysis for the visual detection devices}

A series of experiments has been performed to measure the perception probability of three visual detection devices. We prepared 10 distinct connectors with the guideline, housing hole, and color difference. Each connector was set into two states, full and half engagements, as shown in the Fig. 4. Then, 30 appraisers are inquired to judge intuitively the engagement status in 3 seconds for each sample in a random order. Thus, total 60 trials for each connector have been conducted. Responses from appraisers fall into one of four categories, which are full to full (full engagement state to full engagement decision), full to half, half to full, and half to half. We regarded the success of perception as the right judgment, i.e. full to full and half to half. In preliminary logistic regression analysis, the color difference is not statistically significant, so we exclude it. Table 2 shows the final result of logistic regression analysis for the visual detection devices of the guideline and the housing hole.

Table 2. Logistic regression analysis for visual detection devices after omitting color difference

\begin{tabular}{c|c|c|c|c|c|c}
\hline \hline Source & Coef. & Estimate & Odd ratio & Model & Resid. Dev. & $\operatorname{Pr}(>\operatorname{Chi})$. \\
\hline (Intercept) & $\alpha_{v}$ & 0.4333 & 1.5424 & NULL & 197.03 & \\
\hline Guideline & $\beta_{v, 1}$ & 1.4483 & 4.2558 & Guideline & 177.90 & $1.22 \mathrm{e}-5^{* *}$ \\
\hline Housing hole & $\beta_{v, 2}$ & 0.3156 & 1.3711 & Housing hole & 174.07 & $0.05^{*}$ \\
\hline R-squared & \multicolumn{2}{|c|}{ McFadden- $R^{2}$} & 0.0834 & \multicolumn{2}{c}{ Cragg \& Uhler- $R^{2}$} & 0.6157 \\
\hline
\end{tabular}




\section{Significance: $* *<0.01 ; *<0.1$}

In Table 2, the p-values $(\operatorname{Pr}(>$ Chi.) ) for the Chi-squared test for the guideline and the housing hole is less than 0.01 and about 0.05 , respectively. Therefore, both factors are statistically significant under $95 \%$ significance level. The estimates (Estimate) of the guideline and the housing hole indicates that the use of the two devices increases the log odds of $p_{v}$ as 1.4483 and 0.3156 , respectively. The improvement of the residual deviance (Resid. Dev.) implies that the final model is more suitable than the NULL model. Finally, we can obtain the binary logistic model in Eq. (5), where $x_{\text {guideline }}$ is a Boolean variable, which is 1 if a guideline exists, otherwise, 0 ; $x_{\text {hole }}$ is a Boolean variable, which is 1 if a housing hole exists, otherwise, 0 . In the logistic regression, pseudo- $R^{2}$ such as McFadden- $R^{2}$ and Cragg \& Uhler- $R^{2}$ are used instead of conventional $R^{2}$ to test goodness of fit of the model. The two of pseudo- $R^{2} \mathrm{~s}$ indicate that the visual detection devices are not confident factors. Only $62 \%$ of the response variable variation can be explained by the logistic model. However, it is clear that the visual detection devices affect the perception probability of complete engagement. In addition, as described in Section 4, the proposed stochastic evaluation system automatically adjusts the importance, which can be applied to the evaluation of the feeling of mating to increase accuracy.

$$
p_{v}=\frac{1}{1+e^{-\left(\alpha_{v}+\beta_{v, 1} x_{\text {guideline }}+\beta_{v, 2} x_{\text {hole }}\right)}}
$$

\subsection{Logistic regression analysis for the locking sound}

We performed a series of experiments for a total 18 appraisers to estimate the perception probability for locking sound. The $p_{s}$ depends on the loudness, frequency, timbre, and noise level of circumstance. In this paper, however, we limit our scope only on the loudness level and the noise level due to the limited instrument. The noise can disrupt perception of the locking sound. The procedure of experiments is as follows: First, the recorded factory noise of $80 \mathrm{~dB}$, which is a standard level of common factories, is generated using surround speakers. Next, a group of blinded appraisers stand backward every $30 \mathrm{~cm}$ around the center speaker. Then, a set of recorded locking sounds from $55 \mathrm{~dB}$ to $66 \mathrm{~dB}$ are exposed. Finally, response data from all appraisers about the successes or failures of perception are collected and a logistic regression is performed with the data. The results are summarized in Table 3.

Table 3. Logistic regression analysis for locking sound

\begin{tabular}{|c|c|c|c|c|c|c|}
\hline Source & Coef. & Estimate & Odd ratio & Model & Resid. Dev. & $\operatorname{Pr}(>$ Chi. $)$ \\
\hline (Intercept) & $\alpha_{s}$ & -20.8698 & $8.64 \mathrm{e}-10$ & NULL & 1087.26 & \\
\hline Locking Sound & $\beta_{s}$ & 0.3801 & 1.4624 & Locking Sound & 8.43 & $2.2 \mathrm{e}-16 * *$ \\
\hline R-squared & \multicolumn{2}{|c|}{ McFadden- $R^{2}$} & 0.9345 & \multicolumn{2}{|c|}{ Cragg \& Uhler- $R^{2}$} & 1.0000 \\
\hline
\end{tabular}

In Table 3, the p-value of the locking sound for the Chi-squared test is less than 0.01 , so it is statistically significant with $99 \%$ significance level. The estimate indicates that a unit (dB) increase of locking sound improves the $\log$ odds of $p_{s}$ as 1.4624 . The residual deviance implies that the model including the locking sound fits much better than the NULL model. Finally, we can build the logistic model in Eq. (6) for locking sound, where $l_{\text {sound }}$ is the sound pressure level $(\mathrm{dB})$ of locking sound for a connector. Both of the McFadden- $R^{2}$ and the Cragg \& Uhler- $R^{2}$ are almost to one. It implies that the logistic model accurately describes the data. Fig. 7 shows the scatter plot between the perception probability and the sound pressure level of locking sound, and the logistic regression line by Eq. (6).

$$
p_{s}=\frac{1}{1+e^{-\left(\alpha_{s}+\beta_{s} l_{\text {sound }}\right)}}
$$




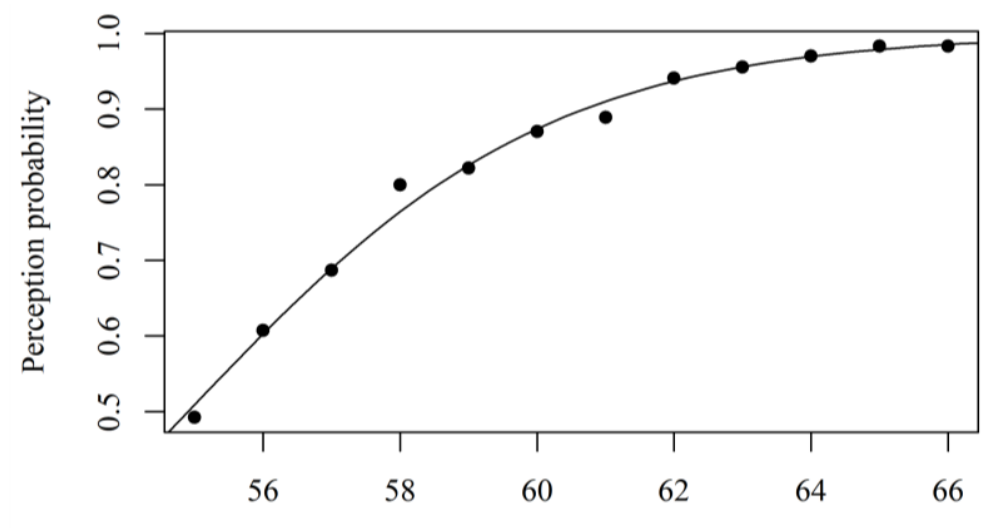

Sound pressure level of locking sound[dB]

Fig. 7 Logistic regression results of perception probability and locking sound

\subsection{Logistic regression analysis for the force drop}

As mentioned earlier, there are four types of insertion force profiles: Type I, II, III, and IV. However, we were forced to exclude the Type II, III, and IV connectors from this experiment. Since the Type II connectors have zero force drop rate, there is no distinct relevance between the force drop rate and the feeling of force drop. The Type III and IV connectors could generate fake feeling caused by the dual force drops, so it can lead to an incorrect conclusion. The experiment proceeded as follows. We have prepared 10 distinct Type I connectors for each of five, i.e. total 50 connectors. The 20 evaluators wore a blindfold, gloves, and earplugs to avoid exogenous effects. In each experiment, we randomly selected 20 connectors and tagged a number to each connector. Then, every tester gauges whether the feeling of force drop existed or not during mating operations. Each evaluator repeated this step five times in random order. Finally, the success or failure of detection for the feeling of force drop was recorded. We measured the force drop rate of each connector by a force gage under the speed of $50 \mathrm{~mm} / \mathrm{min}$ and $200 \mathrm{~Hz}$ sampling rate. The scatter plot of Fig. 8 (left side) shows the correlation of the force drop rate and the success ratio of perception. In the figure, the correlation seems to be weak and the variance of the feeling of force drop is large on even same connector.

Throughout extensive experimentation, we could recognize another factor, the sealing, affecting the feeling of force drop. A sealing connector is a special connector applied for oil or waterproof circumstance. If we separate the connectors into two groups according to sealing, we can draw another scatter plot of Fig. 8 (right side). In the figure, the black circles represent non-sealing connectors, and the white triangles represent sealing connectors. The correlation is effective to only non-sealing connectors. We also found that the variance of the force drop rate is relatively large in even the same model of connectors. The force drop rate changes whenever a mating operation is conducted. It comes from the wearing out of the terminals and the locking part. We measured the insertion force after finishing the above tests owing to a limited number of samples. It causes the significant variance of the force drop rate. Finally, with the collected data, we performed a logistic regression analysis and illustrated the results in Table 4. 

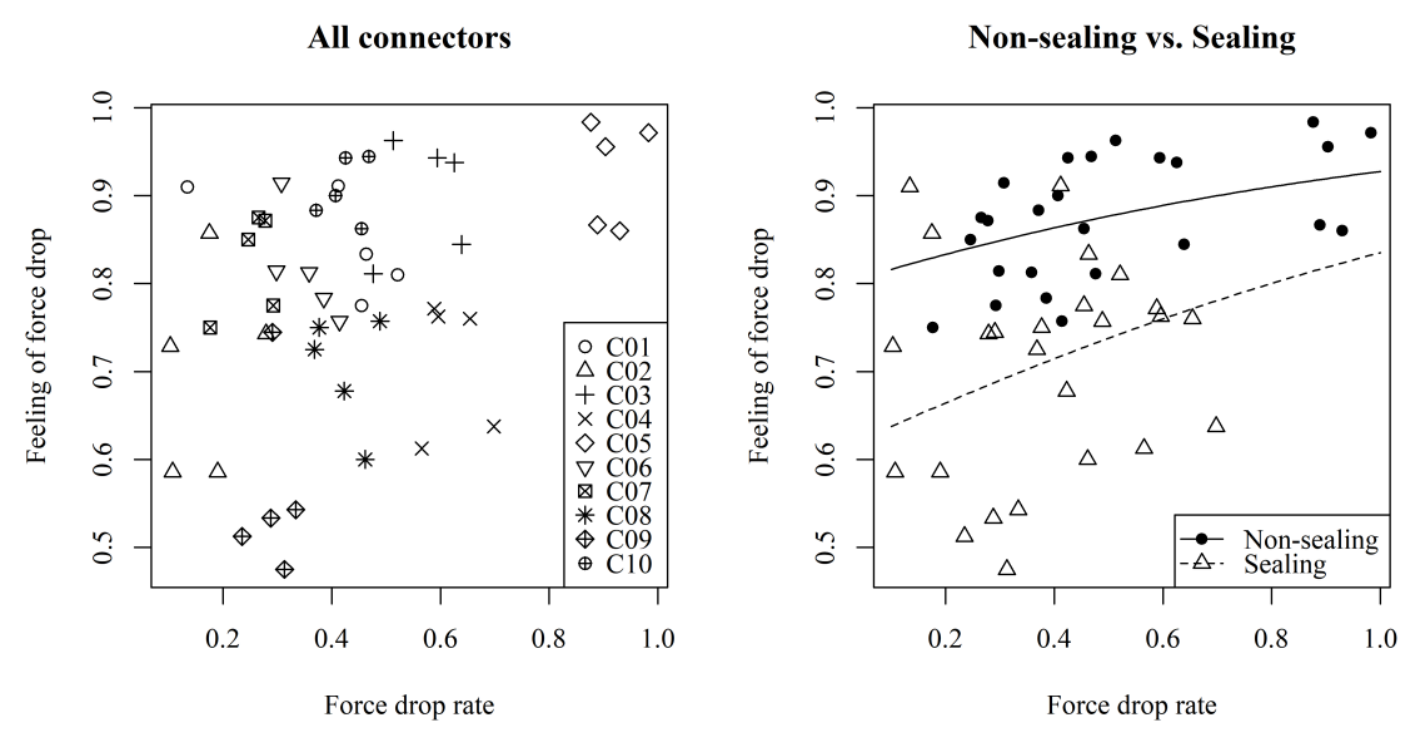

Fig. 8 Scatter plot of feeling of force drop vs. force drop rate

Table 4. Logistic regression analysis for feeling of force drop

\begin{tabular}{c|c|c|c|c|c|c}
\hline \hline Source & Coef. & Estimate & odd ratio & model & Resid. Dev. & $\operatorname{Pr}(>$ Chi. $)$ \\
\hline (Intercept) & $\alpha_{d}$ & 1.3732 & 3.9480 & NULL & 286.15 & \\
\hline Drop Rate & $\beta_{d, 1}$ & 1.1746 & 3.2367 & Drop Rate & 212.87 & $2.2 \mathrm{e}-16^{* *}$ \\
\hline Sealing & $\beta_{d, 2}$ & -0.9259 & 0.3962 & Sealing & 94.10 & $2.2 \mathrm{e}-16^{* *}$ \\
\hline R-squared & \multicolumn{2}{|c|}{ McFadden- $R^{2}$} & 0.5571 & \multicolumn{2}{c}{ Cragg \& Uhler- $R^{2}$} & 1.0000 \\
\hline
\end{tabular}

Significance: $* *<0.01$

In Table 4, the both p-values of the force drop rate and the sealing status for the Chi-squared test are less than 0.01 . Thus, we can conclude that those factors are statistically significant under $99 \%$ significance level. The estimates indicate that a unit increase in force drop rate improves the $\log$ odds of the perception probability as 1.1746 , while application of sealing decreases it as 0.9259 . The residual deviance represents that the force drop rate and the sealing status significantly increase the goodness of fit of the model. The final logistic regression model is depicted in Eq. (7), where $r_{d}$ is the force drop rate of a connector; $x_{\text {sealing }}$ is a Boolean variable, which is 1 if sealing exists, otherwise, 0 . The McFadden- $R^{2}$ is 0.5571 and the Cragg \& Uhler- $R^{2}$ is 1.0000 . Those imply that the proposed logistic model explains the variance of the data quite well. The two lines of Fig. 7 (right-side) show the logistic regression lines by Eq. (7).

$p_{d}=\frac{1}{1+e^{-\left(\alpha_{d}+\beta_{d, 1} r_{d}+\beta_{d, 2} x_{\text {sealing }}\right)}}$

\section{Verification of evaluation approaches}

\subsection{Overall verification procedure}

It is very difficult to measure the feeling of mating as a ratio scale since it is vague and personal difference affects it. Therefore, the following procedure is employed to show the effectiveness of our evaluation approach: 
Table 5. Characteristics of connectors used in experiments

\begin{tabular}{c|c|c|c|c|c|c}
\hline \hline \multirow{2}{*}{$\begin{array}{c}\text { total number } \\
\text { of connectors }\end{array}$} & \multicolumn{2}{|c|}{ insertion force profile } & \multicolumn{2}{c|}{ connector type } & \multicolumn{2}{c}{ visual detection devices } \\
\cline { 3 - 6 } & I & 24 & 13 & 11 & 0 & 6 \\
\hline \multirow{3}{*}{38} & II & 9 & 7 & 2 & 0 & 2 \\
& III & 0 & 0 & 0 & 0 & 0 \\
& IV & 5 & 3 & 2 & 0 & 3 \\
\hline
\end{tabular}

1) We prepared 38 distinct connectors as shown in Table 5. Then, with all connectors, the following parameters are measured: maximum insertion force, insertion force profile, stroke length, force drop rate, and sound pressure level of locking sound.

2) Evaluators have been informed of the purpose, terminologies, and evaluation factors of our experiment.

3) A factory noise up to $80 \mathrm{~dB}$ is generated using surround speakers.

4) According to the feeling of mating, each evaluator tested and categorized the level of feeling of mating into 5 groups and determined the rank of all connector models. The levels and the ranks are reversely coded.

5) The average level and average rank of the feeling of mating for each connector were calculated.

6) By using the average values, the total score by Kubota and Toi (2009) and the sigma level of our proposed approach were calculated.

In this experiment, the evaluators were randomly selected from among the students who participated in the previous experiments. They have a good understanding of the purpose and process of the experiment so that the reliability of the evaluation can be increased. The profiles of the evaluators are summarized in Table 6. We assumed that all workability factors such as hand space, counterpart stiffness, and visibility are in perfect condition.

Table 6. Profiles for the five evaluators

\begin{tabular}{c|c|c|c|c}
\hline \hline Profile & Gender & Age[year] & Height $[\mathrm{cm}]$ & Weight[kg] \\
\hline Evaluator 1 & male & 24 & 173 & 67 \\
Evaluator 2 & male & 23 & 182 & 82 \\
Evaluator 3 & male & 25 & 178 & 75 \\
Evaluator 4 & male & 24 & 175 & 73 \\
Evaluator 5 & male & 27 & 170 & 65 \\
\hline average & & 24.6 & 175.6 & 72.4 \\
\hline
\end{tabular}

\subsection{Rank Correlation among Evaluators}

Firstly, we investigated the Spearman's coefficient of rank correlation to examine the difference among evaluators. The results are summarized in Table 7. According to the correlation analysis, Evaluator 1 has an entirely different standpoint compared to others. The Evaluators 2, 3, 4, and 5 felt similarly regarding the feeling of mating, but the correlation is not that strong. These results imply that the feeling of mating depends on the worker, so large variances are inevitable.

Table 7. Spearman's Coefficient of Rank Correlation among Evaluators

\begin{tabular}{l|c|c|c|c|c}
\hline \hline & Evaluator 1 & Evaluator 2 & Evaluator 3 & Evaluator 4 & Evaluator 5 \\
\hline Evaluator 1 & 1 & -0.1028 & -0.1415 & -0.2292 & -0.2605 \\
Evaluator 2 & & 1 & 0.5597 & 0.3570 & 0.5385 \\
Evaluator 3 & & & 1 & 0.4656 & 0.6612 \\
Evaluator 4 & & & & 1 & 0.1912 \\
Evaluator 5 & & & & & 1 \\
\hline
\end{tabular}

\subsection{Relationship between the average level and the average rank}


The level of feeling of mating is an interval variable, but the rank of the feeling of mating is an ordinal variable; hence it is inappropriate to apply the Pearson's correlation analysis directly. The average values for two variables, however, can have proper properties for it. Fig. 9 shows the scatter plot and the linear regression line for the two averaged variables. Those are linearly correlated. R-squared value is 0.947 . The $\mathrm{p}$-value for the residuals of Shapiro-Wilk normality test is 0.6524 , i.e. normally distributed. Therefore, we can conclude that the average rank of the feeling of mating can be used as a variable for further analysis.

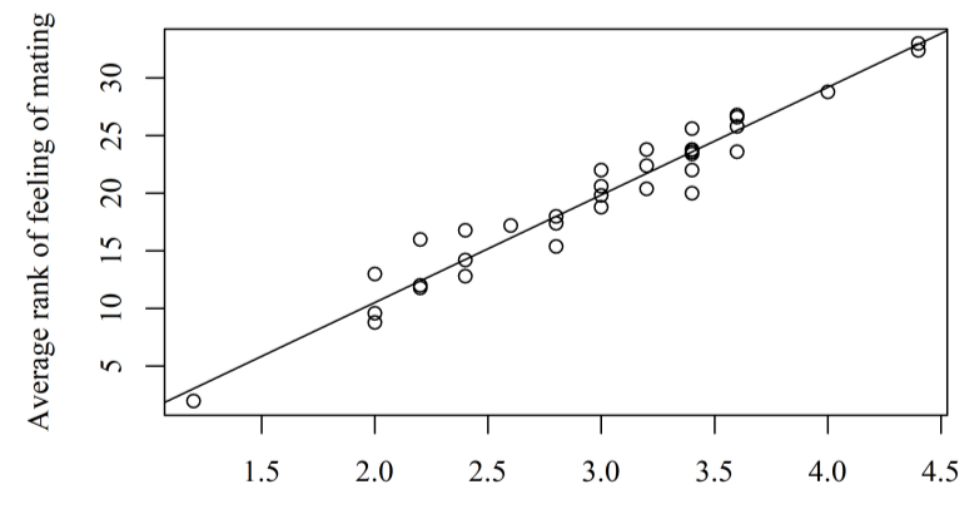

Average level of feeling of mating

Fig. 9 Scatter plot of average level of feeling of mating vs. average rank of feeling of mating for 38 connectors

\subsection{Pearson's correlation coefficient}

To demonstrate the effectiveness of our approach, we conducted Pearson's correlation analyses between the average rank of the feeling of mating and the total score of the Kubota and Toi (2009)'s system (KT), or the sigma level of our proposed system (HJO) with four different cases (Case A, B, C and D). The case A evaluates all 38 connectors with ignoring type II, III, and IV while the case B considers all connector profiles. In the case C, only type I connectors are evaluated and the case D only considers 13 normal (or non-sealing) type I connectors.

\section{- Case A}

In Case A, we counted on all 38 connectors for evaluation. However, our opinion on the profile type II, III, and IV connectors is very pessimistic due to their weak correlation or fake feeling of force drop, so the force drop factor of insertion force profile types II, III, and IV were not considered by setting $t_{d}$ as $[1,0,0,0]$ in Eq. (2). Fig. 10 shows the scatter plots and the linear regression lines. The correlation coefficient of $\mathrm{HJO}\left(r_{H J O}\right)$ is 0.2952 and KT $\left(r_{K T}\right)$ is 0.1903 . The correlation of HJO to the average rank of feeling of mating is a little strong than one of KT, but both are yet weak. Note that the feeling of mating is not measurable because it heavily depends on the perception of a person. Thus, it is very natural that the coefficient is a little low. Therefore, in the following, according to social science decision rule of thumb, we will judge that the correlation is strong if the correlation coefficient is larger than 0.5 , moderate if it is between 0.3 and 0.5 , and weak otherwise (Cohen 1988). 

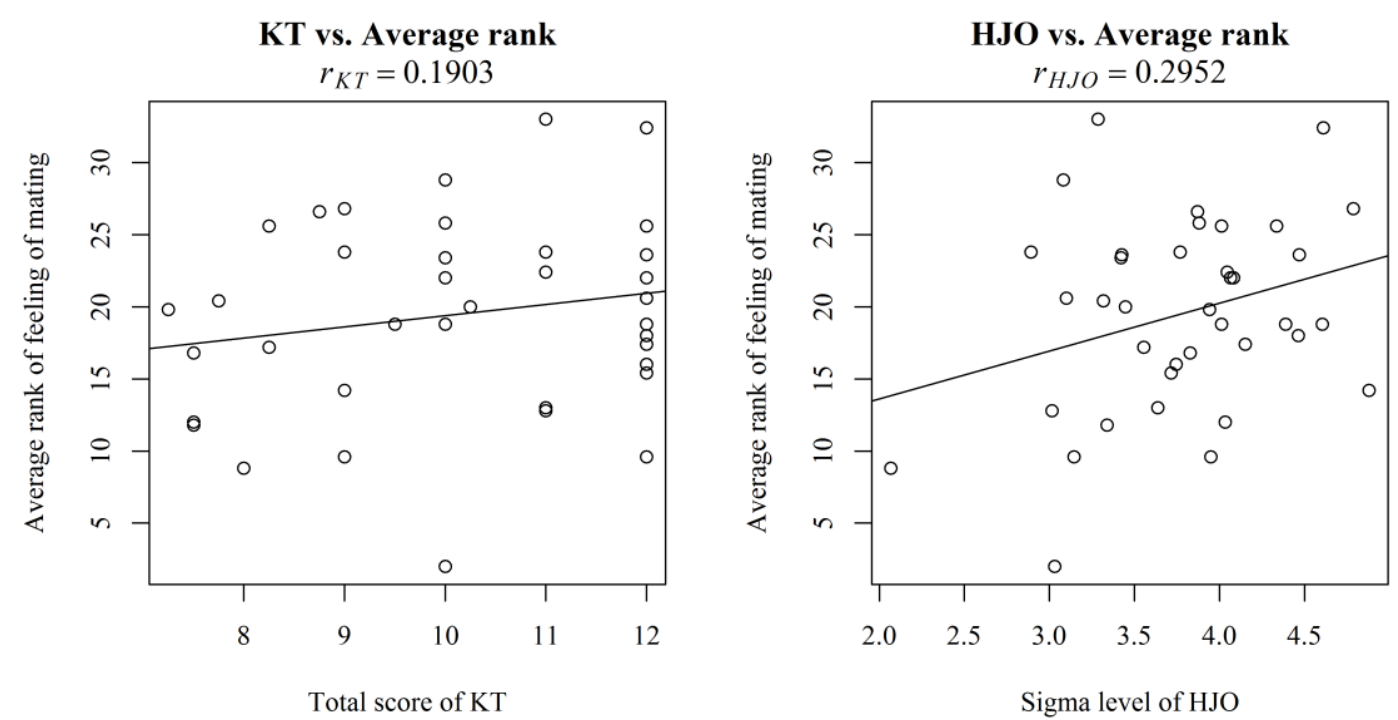

Fig. 10 Scatterplot and regression line of KT and HJO for Case A

\section{- Case B}

Case B considers the force drop factor for all types of insertion force profiles by setting $c_{d}$ as $[1,1,1,1]$. Fig. 11 shows relevant scatter plots and the linear regression lines. In fact, the feeling of force drop exists even if the connector has a zero force drop rate or a dual force drop. As mentioned in Section 3.1, Type III connectors are designed to increase the likelihood of complete engagement, so the feeling of force drop is the strongest if only the mating speed is sufficient. In Case A, we excluded the force drop factor of related connectors because such characteristics could cause half engagement, but in Case B, we included the force drop factor of all types to observe their effects. In Case $\mathrm{B}, r_{H J O}$ increases to 0.4615 . The value is much larger than one in Case A. However, $r_{K T}$ is the same because it does not consider the insertion force profile. This result implies that HJO is more useful if some parameter is adjusted even for Types II, III, and IV.
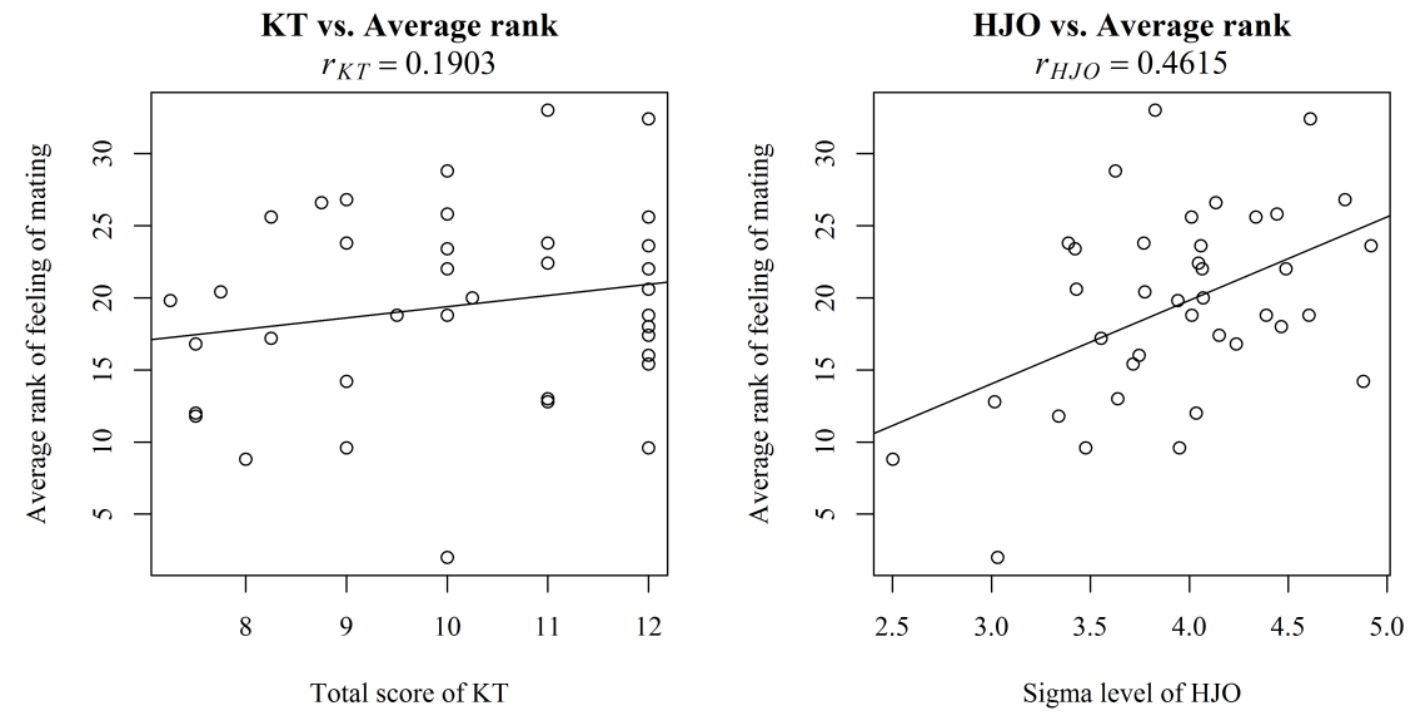

Fig. 11 Scatterplot and regression line of KT and HJO for Case B 


\section{- Case C}

This case is a verification of Case B. Here, we excluded the Type II, III, and IV connectors that cause the low correlation coefficient of the Case A. We only considered 24 Type I connectors without adjusting $c_{d}$. Fig. 12 shows relevant scatter plots and the linear regression lines. The correlations are improved compared to Case B as we expected, since $r_{H J O}$ and $r_{K T}$ are 0.5295 and 0.2334 , respectively.
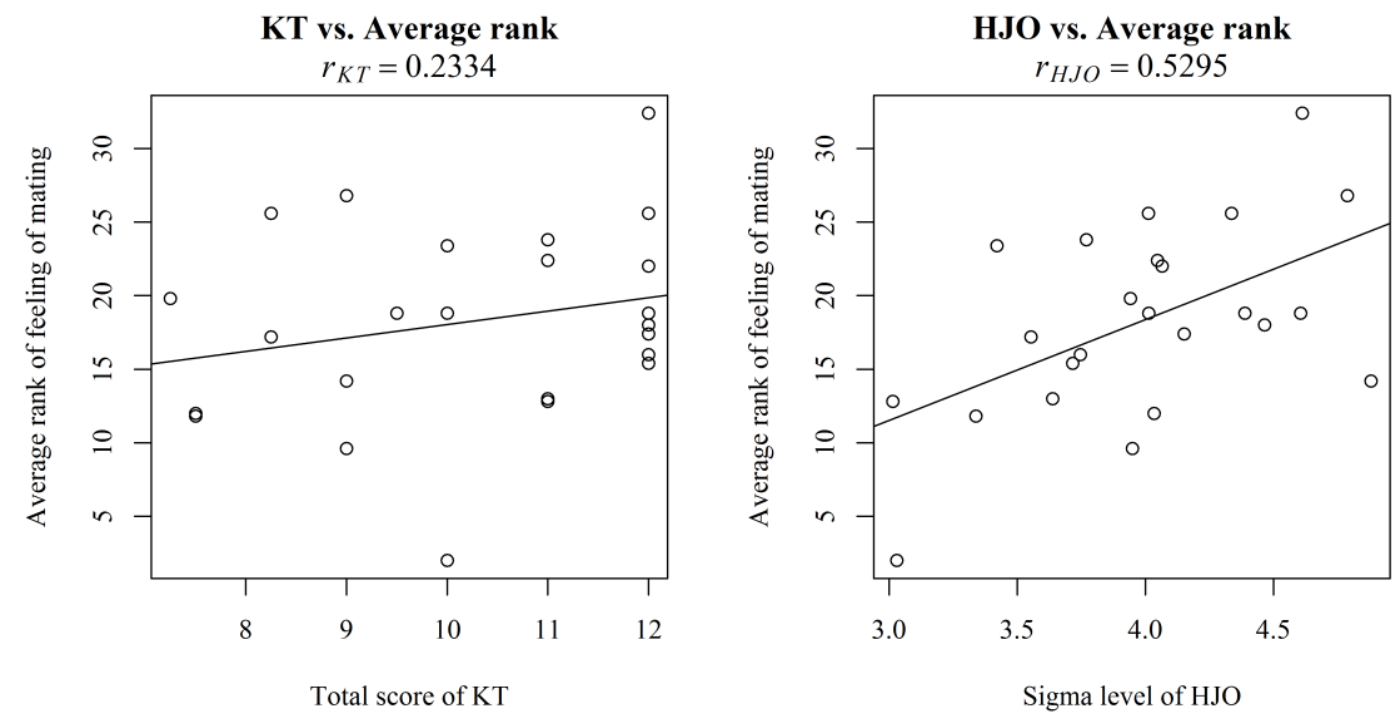

Fig. 12 Scatterplot and regression line of KT and HJO for Case C

\section{- Case D}

This case is designed to conclude our experiments. We excluded the connectors of Types II, III, IV or sealing connectors to eliminate all known exogenous factors. Fig. 13 shows the scatter plots and the linear regression lines. For the 13 selected connectors, $r_{H J O}$ is 0.4925 , which is a little higher than that of Case B but similar to that of Case C. It implies that the logistic regression model in Section 5.3 is effective. The $r_{K T}$ is also slightly improved as 0.2813.
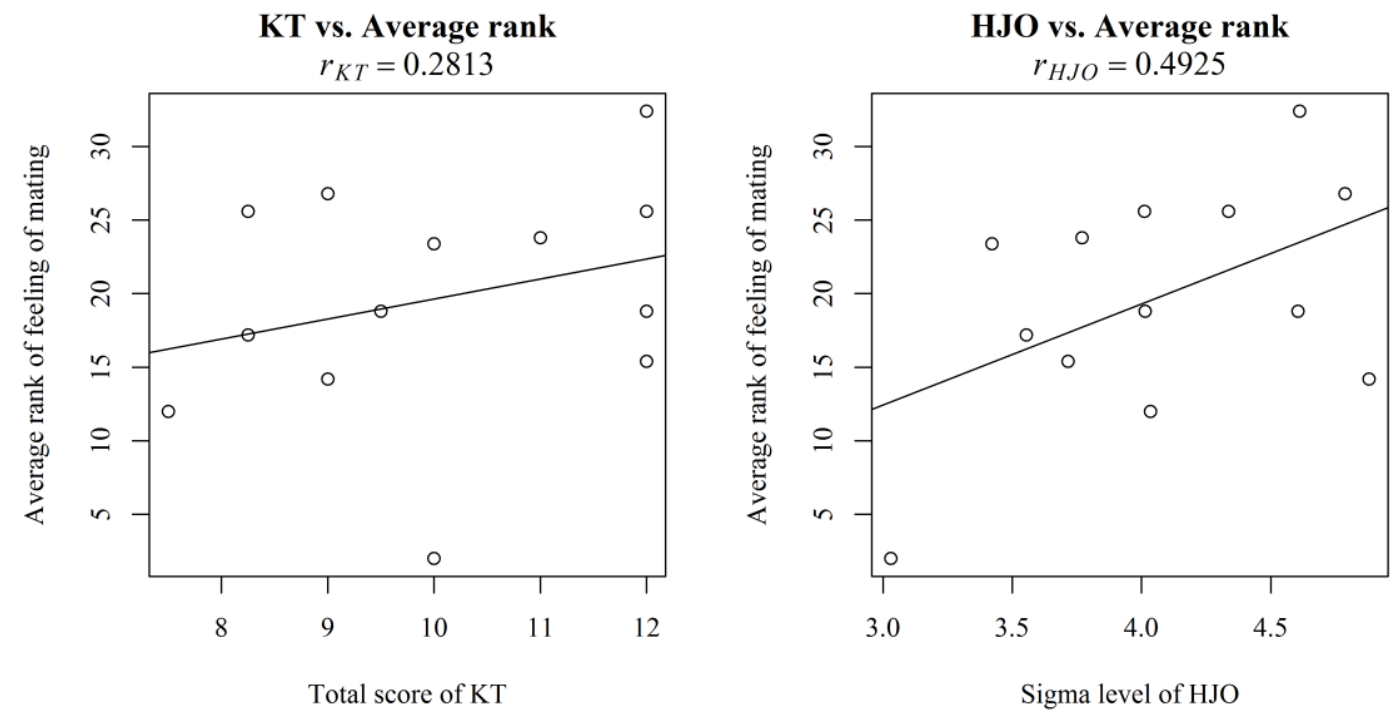

Fig. 13 Scatterplot and regression line of KT and HJO for Case D 
Table 8. Experimental results of correlation analysis

\begin{tabular}{c|c|c|c|c|c|c|c|c}
\hline \hline \multirow{2}{*}{ case } & \multicolumn{4}{|c|}{ considered data } & \multicolumn{2}{c|}{ HJO } & \multicolumn{2}{c}{ KT } \\
\cline { 2 - 9 } & profile & $\begin{array}{l}\text { number of } \\
\text { connectors }\end{array}$ & $c_{d}$ & sealing & $r_{H O}$ & Correlation & $r_{K T}$ & correlation \\
\hline A & All & 38 & {$[1,0,0,0]$} & Both & 0.2952 & Weak & 0.1903 & Weak \\
\hline B & All & 38 & {$[1,1,1,1]$} & Both & 0.4615 & Moderate & 0.1903 & Weak \\
\hline C & I & 24 & {$[1,0,0,0]$} & Both & 0.5295 & Strong & 0.2334 & Weak \\
\hline D & I & 13 & {$[1,0,0,0]$} & Normal & 0.4925 & Moderate & 0.2813 & Weak \\
\hline
\end{tabular}

All results are summarized in Table 8. Throughout the correlation analyses, we could conclude that HJO is an appropriate connector evaluation approach with reasonable sensitivity. On the other hand, KT is not suitable as an assessment tool because the correlation coefficients are low values for all scenarios.

\subsection{Nonparametric Distance measures}

Although we performed linearity and normality tests, it can be issued to use the average rank of the feeling of mating as a variable for Spearman's correlation analysis. In this section, we have cross-checked the superiority of our approach with nonparametric distance measures. If a variable is not in interval or ratio scale and normality is not guaranteed, a nonparametric analysis is preferred. For the purpose, we also verified our approach via nonparametric distance measures (Ok, Lee, and Kumara 2014) such as Spearman's Footrule (D1), Euclidean Distance (D2), Spearman's Rho (D3), Kendall's Tau (D4), and Probabilistic Distance (D5). The distances are calculated from the rank of each evaluator directly. For tied rank, we applied the correction of Becker et al. (1988) instead of the formula in Ok et al. (2014). The calculated distances are summarized in Table 9. The average distances of HJO are smaller than those of KT in all cases except D2. It implies the approach HJO is more appropriate to evaluate feeling of mating as above correlation analysis.

Table 9. Averages of nonparametric distance between variables

\begin{tabular}{c|c|c|c|c|c|c|c|c|c|c|c}
\hline \hline \multirow{2}{*}{ case } & \multirow{2}{*}{ approach } & \multicolumn{4}{|c|}{ the level of feeling of mating } & \multicolumn{4}{c}{ rank of the feeling of mating } \\
\cline { 3 - 13 } & & D1 & D2 & D3 & D4 & D5 & D1 & D2 & D3 & D4 & D5 \\
\hline Case & KT & 316.60 & 121.33 & 0.88 & 0.91 & 0.60 & 228.90 & 88.96 & 0.89 & 0.93 & 0.54 \\
A & HJO & 316.60 & 122.00 & 0.87 & 0.90 & 0.57 & 212.40 & 87.65 & 0.84 & 0.90 & 0.45 \\
\hline Case & KT & 316.60 & 121.33 & 0.88 & 0.91 & 0.60 & 228.90 & 88.96 & 0.89 & 0.93 & 0.54 \\
B & HJO & 315.60 & 121.58 & 0.77 & 0.82 & 0.54 & 198.20 & 81.92 & 0.75 & 0.83 & 0.41 \\
\hline Case & KT & 118.70 & 57.63 & 0.86 & 0.89 & 0.60 & 125.50 & 62.71 & 0.86 & 0.90 & 0.53 \\
C & HJO & 117.90 & 57.56 & 0.70 & 0.77 & 0.52 & 112.40 & 58.48 & 0.69 & 0.78 & 0.39 \\
\hline Case & KT & 28.90 & 19.45 & 0.80 & 0.85 & 0.58 & 92.50 & 60.83 & 0.78 & 0.85 & 0.49 \\
D & HJO & 28.10 & 19.50 & 0.76 & 0.80 & 0.54 & 90.90 & 59.60 & 0.75 & 0.83 & 0.42 \\
\hline
\end{tabular}

* The gray cells indicate lower distance (better correlation).

\section{Discussions and Conclusions}

Through this study, we developed a probabilistic assessment system to quantify the feeling of mating of connectors and verified the accuracy of the assessment using actual electronic connectors. Since the proposed assessment system can express the possibility of half engagement with probability, it helps to make a more reasonable decision than the existing simple assessment system. Through this assessment system, the connectors can be filtered in the design phase prior to mass production. Also, since the magnitude of the influence of each design element change on 
the feeling of mating can be calculated numerically, a direction for improving the connector can be suggested. As a result of the empirical test, the proposed assessment system proved to be suitable for measuring the feeling of mating.

Some of the issues discussed in this study are as follows. First, the correlation between the feeling of force drop and the feeling of mating was not critical. This is significantly different from what we expected at the start of the study. The feeling of force drop is determined by various factors such as the magnitude of the force drop, the force drop rate, the maximum insertion force, the stroke length, and the structure and material of the connector. We have attempted to find a strong correlation between these various factors or their combinations and the feeling of force drop or feeling of mating. However, there were no statistically significant correlations among the measurable factors except the force drop rate. If the relationship between these variables is revealed through additional experiments and data, we expect to build a more reliable assessment system. Second, the connector's locking sound has a different tone depending on the combination of loudness, frequency, and timbre. Some locking sounds sound more vivid despite large circumstance noise. We have tried to clarify the relationship between them through the frequency analysis of the collected locking sounds. However, due to the low resolution of the detection equipment and the lack of knowledge and time, no significant results were obtained other than loudness. If further study is possible, it is very likely to reveal a meaningful relationship between them. Third, visual detection devices are only valid if the worker observes carefully during the mating operation. They allow the worker to be assured that the connector is in full engagement. However, since they are mostly small in size, they require additional observation. Visual detection generally plays a key role in inspection. Therefore, adding a device that allows the worker to easily identify the full engagement on the connector can have a positive effect.

The probabilistic evaluation system proposed in this study can easily add new factors while maintaining the relationship structure and evaluation structure of existing factors. The above discussions can be further improved through design, experimentation, analysis, and modeling. Future studies are planned to build a more reliable assessment system by adding these factors.

\section{References}

Becker, R. A. Chambers, J. M. and Wilks, A. R., The New S Language: A Programming Environment for Data Analysis and Graphics. Monterey (1988), Wadsworth and Brooks/Cole Advanced Books \& Software.

Cohen, J., Statistical Power Analysis for the Behavioral Sciences 2nd ed. (1988), New York: Lawrence Erlbaum Associates.

Denton, T., Automobile Electrical and Electronic Systems 5th ed. (2017), London and New York: Routledge Taylor \& Francis Group.

Elsayed, E. A., Reliability Engineering (2012), John Wiley \& Sons.

Ha, C., and Way, K., Initial Allocation Compensation Algorithm for Redundancy Allocation: The Scanning Heuristic, IIE Transactions, Vol. 40 (2008), pp.678-689.

Huang, J., Di, P. Fukuda, T. and Matsuno, T., Robust Model-Based Online Fault Detection for Mating Process of Electric Connectors in Robotic Wiring Harness Assembly Systems, IEEE Transactions on Control Systems Technology Vol.18, No.5 (2010) pp.1207-1215.

Huang, J., Toshio Fukuda T., and Takayuki Matsuno, Model-Based Intelligent Fault Detection and Diagnosis for Mating Electric Connectors in Robotic Wiring Harness Assembly Systems, IEEE/ASME Transactions on Mechatronics Vol.13, No.1 (2008). pp.86-94.

Kashiyama, M., Half-Fitting Prevention Connector, US Patent 5,938,470 (1999).

Kashiyama, M. and Suzuki, Y, Connector with Slider Detecting Half-Engagement, US Patent 6,752,648 B2 (2004).

Kozono, S. and Toshinori Yamamoto T., Engagement Detecting Structure in Connector, US Patent 6,908,329 (2005).

Kubota, S., and Toi T., In-Process Quality Assurance of Wire Harness Connector Mating, Mazda Technical Review Vol. 27, pp.169-174.

Kuo, W. and Zuo, M. J., Optimal Reliability Modeling: Principles and Applications 1st ed.(2003), John Wiley \& Sons. Lee, K. and Ha, C., Defective Rate Reduction of Connector Mating, ANQ Congress (2014), pp.1-5, Singapore.

Leen, G., and Heffernan D., Expanding Automotive Electronic Systems, Computer Vol.35, No.1 (2002), pp.88-93.

Menard, S., Applied Logistic Regression Analysis 2nd ed. (2002), Thousand Oaks: SAGE Publications.

Mroczkowski, R. S., Electronic Connector Handbook: Theory and Applications (1988), New York: McGraw-Hill.

Ok, C., Lee, S. and Kumara, S., Group Preference Modeling for Intelligent Shared Environments: Social Welfare beyond the Sum, Information Sciences Vol. 278 (2014), pp.588-598.

Pyzdek, T. and Keller, P., The Six Sigma Handbook: Revised and Expanded (2014), New York: McGraw-Hill.

Yagi, S., Tsuji, M. Jinno, K. and Yoneda T., Connector Having Engagement Detecting Device, US Patent 5,618,201 (1997). 